\title{
Photon energy (8-250keV) response of optically stimulated luminescence: Implications for luminescence geochronology
}

\author{
Guerin, Guillaume; Mihailescu, Liviu-Cristian; Jain, Mayank
}

Published in:

Journal of Luminescence

Link to article, DOI:

10.1016/j.jlumin.2018.07.047

Publication date:

2018

Document Version

Peer reviewed version

Link back to DTU Orbit

Citation (APA):

Guerin, G., Mihailescu, L-C., \& Jain, M. (2018). Photon energy (8-250keV) response of optically stimulated luminescence: Implications for luminescence geochronology. Journal of Luminescence, 204, 135-144. https://doi.org/10.1016/j.jlumin.2018.07.047

\section{General rights}

Copyright and moral rights for the publications made accessible in the public portal are retained by the authors and/or other copyright owners and it is a condition of accessing publications that users recognise and abide by the legal requirements associated with these rights.

- Users may download and print one copy of any publication from the public portal for the purpose of private study or research.

- You may not further distribute the material or use it for any profit-making activity or commercial gain

- You may freely distribute the URL identifying the publication in the public portal 


\section{Author's Accepted Manuscript}

Photon energy $(8-250 \mathrm{keV})$ response of optically stimulated luminescence: Implications for luminescence geochronology

Guillaume Guérin, Liviu-Cristian Mihailescu, Mayank Jain

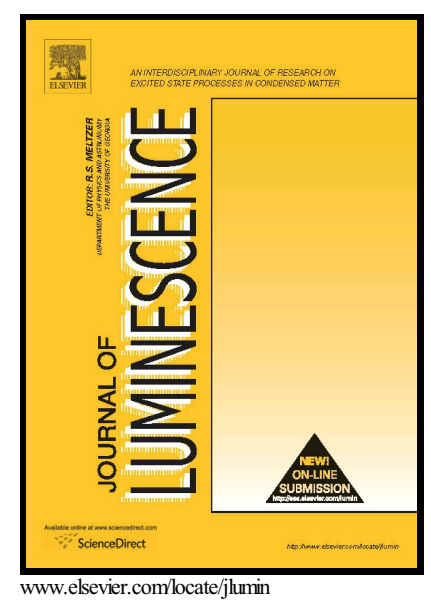

PII: $\quad$ S0022-2313(18)30470-8

DOI: $\quad$ https://doi.org/10.1016/j.jlumin.2018.07.047

Reference: LUMIN15794

To appear in: Journal of Luminescence

Received date: 14 March 2018

Revised date: 21 June 2018

Accepted date: 28 July 2018

Cite this article as: Guillaume Guérin, Liviu-Cristian Mihailescu and Mayank Jain, Photon energy $(8-250 \mathrm{keV})$ response of optically stimulated luminescence: Implications for luminescence geochronology, Journal of Luminescence, https://doi.org/10.1016/j.jlumin.2018.07.047

This is a PDF file of an unedited manuscript that has been accepted for publication. As a service to our customers we are providing this early version of the manuscript. The manuscript will undergo copyediting, typesetting, and review of the resulting galley proof before it is published in its final citable form. Please note that during the production process errors may be discovered which could affect the content, and all legal disclaimers that apply to the journal pertain. 
Photon energy $(8-250 \mathrm{keV})$ response of optically stimulated luminescence: Implications for luminescence geochronology

Guillaume Guérin $^{1,2}$, Liviu-Cristian Mihailescu ${ }^{3}$ Mayank Jain ${ }^{2}$

${ }^{1}$ Institut de Recherche sur les Archéomatériaux, UMR 5060 CNRS - Université Bordeaux Montaigne, Centre de Recherche en Physique Appliquée à l'Archéologie (CRP2A), Maison de l'archéologie, 33607 Pessac cedex.

${ }^{2}$ Center for Nuclear Technologies, Technical University of Denmark, DTU Ris $\varnothing$ Campus, DK-4000 Roskilde, Denmark.

${ }^{3}$ SCK-CEN, Belgian Nuclear Research Centre, Boeretang 200, 2400 Mol, Belgium.

Corresponding author: G. Guérin (guillaume.guerin@u-bordeaux-montaigne.fr)

\section{Abstract:}

Understanding luminescence response of dosimeters and scintillators to ionising radiation is an important yet poorly understood area in the field of solid state dosimetry. Such an understanding is particularly critical for quantifying effective dose (rates) in wide-energy mixed-radiation fields. While there have been several investigations on characterising the ionising- energy response of artificial dosimeters (e.g. $\mathrm{LiF}, \mathrm{Al}_{2} \mathrm{O}_{3}: \mathrm{C}$, etc.), there exist no studies on natural dosimeters such as quartz and feldspar which are extensively used in luminescence geochronology and retrospective dosimetry. Currently, luminescence geochronology assumes that all beta and gamma irradiations in nature (from the decay of $K$, the $U$ and Th radioactive chains) are equivalent in terms of luminescence production per unit dose to each other, and to the laboratory ${ }^{137} \mathrm{Cs},{ }^{60} \mathrm{Co}$ (gamma) and ${ }^{90} \mathrm{Sr} /{ }^{90} \mathrm{Y}$ (beta) irradiations used for calibration and/or equivalent dose determination. In this study, we experimentally determine the quartz Optically Stimulated Luminescence (OSL) and feldspar Infra-Red Stimulated Luminescence (IRSL: IRSL at $50^{\circ} \mathrm{C}$ and post-IR IRSL at $290^{\circ} \mathrm{C}$ ) response to irradiations with photons of variable energies $(8-250 \mathrm{keV})$. We show that microdosimetric effects lead to a steady, significant decrease in quartz OSL production efficiency for $<80 \mathrm{keV}$ photons. Similar to quartz, the local saturation of the electron traps also lead to a systematic decrease in luminescence efficiency for $<120 \mathrm{keV}$ photons in feldspar; however, this effect competes with an increase in recombination efficiency due an increased number density of hole centres in the vicinity of the trap, thus giving a peak-shaped IRSL efficiency response in the range of 250 to $8 \mathrm{keV}$. Our results suggest that dose-rate 
conversion factors, specific to radiation type and radioisotope, should be used for age calculation, and significant care should be taken for luminescence based calibration of the dose rate of the laboratory irradiation source.

Key-words: Luminescence dating; x-ray efficiency; ionisation density effects.

\section{Introduction}

Optically stimulated luminescence (OSL) and thermoluminescence (TL) are widely used in retrospective (passive) dosimetry for wide ranging applications in environment, health and geochronology. OSL or TL dosimetry relies upon calibration of the signal induced from trapped charges (electrons and holes) in a material to dose absorbed $\left(\mathrm{Gy}=J \cdot \mathrm{kg}^{-1}\right)$ from exposure to ionising radiation. In order for this calibration to be accurate, it is imperative that any variations in the ability of different ionising radiations to trap charge in the material (dosimeter) are accounted for. In geochronology, the focus of this article, the age of a sediment sample is obtained by dividing the equivalent dose (Gy) in quartz or feldspar mineral grains by the ambient dose rate (Gy.s $\mathrm{s}^{-1}$, or more commonly Gy. $\left.\mathrm{ka}^{-1}[1]\right)$ in sediment. Here equivalent dose is defined as the laboratory dose that results in the same luminescence intensity as the natural signal (e.g [2]). It is assumed that the luminescence resulting from unit dose is independent of the sources of ionising radiation (beta or gamma rays), whether in nature or in the laboratory. This assumption does not apply to alpha radiation because of its high Linear Energy Transfer (LET) resulting in trap saturation effects around the alpha track in the crystal. In contrast, the beta, gamma and cosmic rays (see, e.g., [1]) deposit their energy more uniformly within the crystal. Two solutions are commonly employed to address the dosimetry of alpha particles: a) chemical etching to remove the alpha irradiated outer layer of the dosimeter (e.g. sand sized grains of quartz), or b) accounting for the efficiency of alpha particles to induce luminescence (alpha efficiency) in the calculation of an effective dose rate (e.g. dating of silt sized grains [3]; heated stones [1]).

The assumption that beta and gamma radiations are equivalent is not completely true; it has been shown for example that low energy $\mathrm{x}$-rays are not as efficient as ${ }^{137} \mathrm{Cs}$ gamma rays because they produce higher ionisation density per unit dose through energy absorption by creation of photoelectrons [4]. Thus, the assumption of equivalence must fail for both low energy photons and electrons (since ultimately ionisation induced by photon irradiations is the result of secondary 


\section{ACCEPTED MANUSCRIPT}

electron interactions in the crystal). Given the implications of this understanding for retrospective dosimetry and dating, it is imperative that a detailed study of the photon or electron energy response be undertaken. Understanding the electron energy response is especially important for dosimetry since irrespective of the primary radiation (beta, gamma or cosmic) most of the dose is deposited by secondary electrons.

A direct examination of the luminescence response to low energy electrons (e.g. $<50 \mathrm{keV}$ ) is difficult because of their high LET and short range; typical quartz grains of $150 \mu \mathrm{m}$ diameter are too large to do such investigations. However, this problem can be circumvented by using photons instead. Unlike electrons, the mean free path of photons even for energies of tens of keV is larger than the mean dimension of sand sized quartz grains. Moreover, at low energies ( $<50 \mathrm{keV})$ the dominant mechanism of interaction is photo-electric effect; thus an electron energy response should largely mimic a photon energy response below $50 \mathrm{keV}$. Thus, studying the photon energy response gives directly the information on both the response to the photons as well as to the low energy electrons.

The photon energy response has been studied for various detectors, e.g. LiF [5,6], $\mathrm{BeO}$ [7], $\mathrm{Al}_{2} \mathrm{O}_{3}$ $[8,9]$. The focus of many of these studies has been to measure an overall energy-response function which is of direct practical consequence in medical dosimetry, and arises from a combination of energy absorption coefficient (in general the reference quantity is dose to air, or to water) and ionisation density effects influencing the signal emitted by the dosimeter (usually TL or OSL). Our study aims at measuring and understanding the photon energy-response curve of quartz and Kfeldspar, and testing the assumption that cosmic, beta and gamma dose rates can be considered equivalent for estimation of dose rate in natural environments. This study has important bearing for environmental dosimetry using natural materials and luminescence (OSL, TL) dating, as well as for the use of reference laboratory $\mathrm{x}$-ray sources for dose calibration using these materials.

\section{Samples and luminescence measurements}

\subsection{Luminescence measurement conditions}

Grains were mounted in $9 \mathrm{~mm}$ base diameter stainless steel cups using silicone oil. Aliquots of $\sim 3 \mathrm{~mm}$ in diameter, comprising several hundreds of grains, were measured for both quartz and feldspar extracts. Luminescence measurements were made using a Ris $\varnothing$ TL/OSL DA-20 reader. For quartz, blue (470 $\mathrm{nm}$ ) light-emitting diodes (LED) were used with $7.5 \mathrm{~mm}$ Hoya U-340 detection filters. For feldspar, IR diodes emitting at $875 \mathrm{~nm}$ were used in combination with coupled Schott BG39 and Corning 7-59 detection filters (transmission 320-460 nm [10,11]). The signal from the first $0.5 \mathrm{~s}$ of stimulation was summed to estimate the OSL signal intensity; the background was estimated from the following $1 \mathrm{~s}$ (early background subtraction). For Infra-Red Stimulated Luminescence (IRSL) 


\section{ACCEPTED MANUSCRIPT}

signals, we used the first $4 \mathrm{~s}$ and last $20 \mathrm{~s}$ for signal and background estimation, respectively. A Single-Aliquot Regenerative-dose procedure (SAR: [12]) was used for all dose measurements, using a test dose of $1 \mathrm{~Gy}$; the SAR protocol compensates for any sensitivity changes in the measurement of the luminescence dose response curve by using a test dose response. Equivalent dose is estimated by interpolating sensitivity corrected luminescence intensity (induced from $\mathrm{x}$-ray or gamma) on the sensitivity corrected luminescence dose response curve measured using a reference laboratory beta source.

\subsection{Samples}

Table 1 presents the list of samples selected in this study. These samples were selected following several criteria. Firstly, because we are interested in dating applications, the quartz OSL signal had to be dominated by the fast component to avoid any undesirable effect of the slow components $[21,22,23]$. Then, to avoid saturation effects, we worked in the dose range where the luminescence signal can be approximated to grow linearly with dose ( $<5 \mathrm{~Gy}$ for quartz, $<10 \mathrm{~Gy}$ for Kfeldspar). Finally, we aimed for sensitive samples to obtain high precision; typically, we wanted doses of $1 \mathrm{~Gy}$ to be easily measured with a good reproducibility. To test this, we carried out various beta and gamma dose recovery tests on several samples, which were first bleached in a solar simulator SOL2 for 3 hours, and then given a dose of about $1 \mathrm{~Gy}$ and measured using a SAR protocol [12].

A total of seven quartz samples were selected for studying the OSL response as a function of photon energy. These samples came from a range of geographic locations and geomorphologic settings (Table 1) to reflect - even though only partially - the diversity of samples encountered in the field of luminescence dating. Only three of these samples were deemed bright enough and yielded measured-to-given dose ratios consistent with unity at two standard errors: Røm $\varnothing$ - B5 [13], 82105 [14], both unheated sediment, and 031301 [15] from heated ceramics. The remaining four samples were sensitised by heating them in an oven, at a temperature of $500^{\circ} \mathrm{C}$ held for $30 \mathrm{~min}$. It was shown that the response to low energy $\mathrm{x}$-rays is sample dependent to a much bigger extent than that to gamma radiation [24]; thus, we expected that this sample-to-sample variability in the response to $x$ rays will be appropriately covered in our sample selection.

In addition to these seven quartz samples, we also selected potassium feldspar extracts from two unheated sediment samples to study the post-IR IRSL at $290^{\circ} \mathrm{C}$ (PIRIR 290 : [25]) dependence on photon energy. These two samples were thermally annealed and sensitised (like the quartz extracts) prior to $x$-ray irradiations, and then their $\mathrm{plRIR}_{290}$ characteristics tested in a dose recovery experiment. We also looked at the IRSL at $50^{\circ} \mathrm{C}\left(\mathrm{IR}_{50}\right)$ signal from the pIRIR $\mathrm{R}_{290}$ protocol; both signals gave good dose recovery ratio. Table 1 summarises the list of samples studied: in particular, result of 


\section{ACCEPTED MANUSCRIPT}

dose recovery tests are provided. Fig. 1 shows dose recovery ratio values against intensity of first test dose response, and thus gives an indication of the relative samples brightness.

\section{Irradiation set-up}

The aim of this study is to compare the luminescence efficiency of $x$-rays as a function of their energy. Our reference radiation is ${ }^{137} \mathrm{Cs}$ gamma radiation (662 keV) at the DTU Nutech, which is commonly used to calibrate the ${ }^{90} \mathrm{Sr} /{ }^{90} \mathrm{Y}$ beta source in luminescence dating [26]. The ${ }^{137} \mathrm{Cs}$ source has a secondary calibration using a standardised ionisation chamber by the Statens Institut for Strålehygiejne (Danish State Institute for Radiation Hygiene). The air kerma measured through ionisation chamber at a specific distance from the source is converted to dose in quartz using the appropriate mass absorption coefficients. The absorbed dose in quartz has been independently verified by Fricke dosimetry [27]. There is a general understanding that the OSL response of quartz to the high energy gamma $\left({ }^{137} \mathrm{Cs}\right)$ and beta $\left({ }^{90} \mathrm{Sr} /{ }^{90} \mathrm{Y}\right)$ radiations is the same, thus the calibration of the beta source based on OSL measurements does actually reflect the true dose rate of the ${ }^{90} \mathrm{Sr} /{ }^{90} \mathrm{Y}$ source.

X-ray irradiations were performed at the Laboratory for Nuclear Calibrations (LNK) of SCK.CEN, the Belgian Nuclear Research Centre, with the reference narrow (' $N$ ') spectrum series as defined by the ISO 4037-1 specification [26]. Two x-ray tubes were used for this study (100 kV and $300 \mathrm{kV}$ acceleration potentials, respectively) to cover the range 10-300 kV. The $\mathrm{x}$-ray beams are calibrated in terms of kerma in air $\left(\mathrm{K}_{\mathrm{air}}\right)$ ensuring the traceability to the international standard. The recommendations of the ISO 4037-1 specification [26] were followed during the irradiations. For our study, the important physical quantity is the actual dose delivered to quartz and feldspar grains. This absorbed dose differs from $\mathrm{K}_{\text {air }}$ due to mass absorption coefficients, but also due to attenuation of radiation by the light-tight containers in which the grains were placed, and to non-homogeneous dose deposition within the grains. As a result, calculations are not straightforward. To tackle this, we performed Monte Carlo simulations of the x-ray beams and their interactions with air on the one hand, and quartz/feldspar grains inside our plastic containers on the other. Fig. S1 shows the containers on the holder placed in front of the beam. For each irradiation, the samples were placed in the same position: from left to right, in the top row samples $\mathrm{R} \emptyset \mathrm{m} \emptyset-\mathrm{B} 5,082105$ and 031301, in the middle row samples 981003, 133001 and 962523, and in the bottom row 065414, 963602 and 133075. Finally, for each sample, one sample holder was not irradiated by $\mathrm{x}$-rays; it was used as a control sample to measure dose acquired during transport and storage prior to measurement.

\section{Delivered doses: GEANT4 simulations}


The $x$-ray spectra of the narrow series are defined by ISO specifications in terms of experimental quantities, such as mean energy, resolution, and half value layers. However, the shape of these spectra varies from one tube potential to another, and there is no mathematical function describing each spectrum. To overcome this problem and to have confidence in our simulation results, we reproduced the narrow series spectra using ISO specifications using the GEANT4 radiation transport toolkit (version 4.9; $[29,30]$ ). The transport of electrons of fixed energies (one energy value per tube potential) was simulated inside a $1 \mathrm{~mm}$ thick Beryllium tube (this corresponds to the inherent filtration as recommend by ISO 4037-1) containing vacuum and directed towards a Tungsten target oriented at $45^{\circ}$ with respect to the electron beam. Electromagnetic interactions were simulated using the Penelope datasets [31]. The energy of $x$-rays passing through the $1 \mathrm{~mm}$ Beryllium window and the filter combination, as specified by ISO recommendations was recorded in the simulation, and example spectra are shown in Fig. 2. Table 2 lists the characteristics (mean energy and resolution) of each spectrum as obtained by these simulations, in comparison with the ISO 40371 specifications. The latter recommend that the mean energy must be within $\pm 5 \%$ of the specified value, and the resolution of $\pm 15 \%$. Our simulated $x$-ray spectra fall within these intervals for all tube potentials, and as a result we can consider them to be reliable for the simulation of our irradiations with narrow-spectra series.

These spectra were then used as input data to simulate the irradiations of quartz and feldspar grains. To ensure a good control of the dose delivered to the grains, a single layer of grains was deposited on double-sided tape in the light-tight containers (Fig. S1 shows the experimental setup for the irradiations). The lid thickness, facing the beam, was measured with a calliper (600 $\mu \mathrm{m})$, as well as the density of the plastic $\left(1.2 \mathrm{~g}_{\mathrm{cm}} \mathrm{cm}^{-3}\right.$, based on the mass and volume increase in a water containing Beaker in which 100 containers were added). These values were used in the GEANT4 simulations: for each narrow spectrum, a parallel beam of x-rays was directed at a light tight container - in the simulations, quartz and feldspar grains (with a diameter of $200 \mu \mathrm{m}$ ) were sandwiched between two layers of plastic representing the faces of the containers (as a result, although negligible, any back-scatter is also simulated). Fig. 3 shows the dose delivered to quartz and feldspar grains during the irradiations corresponding to 1 Gy kerma in air (see also Table 3). The uncertainties appearing in this Table and Figure correspond to the quadratic sum of the GEANT4 simulation uncertainties and the dose rate calibration uncertainties on $\mathrm{K}_{\mathrm{air}}$ values; the overall uncertainty might be underestimated, since container thickness and density have not been considered in the uncertainty budget.

For low energies, where the photoelectric effect is the dominant interaction process between electrons and mineral grains, the difference in the average atomic number between quartz 


\section{ACCEPTED MANUSCRIPT}

and K-feldspar leads to great differences in energy absorption; for example, the photoelectric cross section for photons of energy $50 \mathrm{keV}$ is $0.12 \mathrm{~cm}^{2} . \mathrm{g}^{-1}$ for quartz compared to $7.5 \mathrm{~cm}^{2} \cdot \mathrm{g}^{-1}$ for K-feldspar. As a result (Fig. 3, Table 3), the doses deposited with low energy photons is much bigger in K-feldspar than in quartz (doses of up to $\sim 5$ Gy delivered to quartz grains, and 8 Gy to K-feldspar grains).

\section{OSL and pIRIR 290 efficiency of photons as a function of x-rays energy}

The relative efficiency of a particular photon source in inducing luminescence can be estimated using the formula:

$$
\xi_{\mathrm{h} v \rightarrow \gamma \operatorname{Cs} 137}=\frac{S_{X-r a y}}{S_{C s-137}},
$$

where $\xi$ is the efficiency of the $\mathrm{x}$-ray source with respect to the ${ }^{137} \mathrm{Cs}$ gamma radiation in producing an OSL signal, and $S$ is the sensitivity, i.e. the OSL response per unit dose specific to a particular radiation.

For each $\mathrm{x}$-ray irradiation, one can estimate, in a robust manner using the single aliquot regenerative dose protocol (SAR; [12]), the beta equivalent dose $E D_{X-\text { ray }}$, defined as the beta dose $\left({ }^{90} \mathrm{Sr}_{-}{ }^{90} \mathrm{Y}\right)$ resulting in the same luminescence intensity as the given $\mathrm{x}$-ray dose $\left(D_{X-\text { ray }}\right)$. For the approximate linear part of the dose-response curve:

$D_{X-\text { ray }} \cdot S_{X-\text { ray }}=E D_{X-\text { ray }} . S_{S r 90-Y 90}$.

Similarly, for the gamma ${ }^{137} \mathrm{Cs}$ irradiation one can write:

$D_{C S-137} \cdot S_{C s-137}=E D_{C S-137} \cdot S_{S r 90-Y 90}$

Using the above two equations and substituting them in (1) gives:

$$
\xi_{\mathrm{h} \nu \rightarrow \gamma \operatorname{Cs} 137}=\frac{\frac{E D_{X}-\text { ray }}{D_{X-\text { ray }}}}{\frac{E D_{C s-137}}{D_{C s-137}}} .
$$

Here $D_{X \text {-ray }}$ is estimated from GEANT4 modelling and $K_{\text {air }}$ and $D_{C s-137}$ is estimated based on the gamma irradiation and calibration procedure used at DTU Nutech [26]. $E D_{X-\text { ray }}$ and $E D_{c s-137}$ are based on the SAR protocol using the reference beta source in the Ris $\varnothing$ TL-OSL reader. Because of the proven strength of the SAR protocol, and the possibility to do internal tests of accuracy (such as the dose recovery tests, etc.), we used equation (2) based on equivalent dose measurement rather than equation (1) based on sensitivity measurement $(S)$, to estimate $\xi$. It should be emphasised here that, since our results are normalised to absorbed dose (rather than to photon flux), the effects of varying 


\section{ACCEPTED MANUSCRIPT}

photon interaction cross sections and mass energy absorption coefficients are already taken into account in $\xi$. In other words, any deviation of $\xi$ from unity reflect microdosimetric effects.

\subsection{Quartz OSL results}

Fig. $4 a$ and Table 4 (see also Table S1) show the efficiency of $x$-rays as a function of the photon mean energy for quartz OSL. For each sample (see legend, Fig. 4a), the efficiency is estimated from the weighted mean of 5 aliquots. The filled black circles represent the arithmetic mean of the seven samples with the associated $1 \sigma$ standard error of the mean plotted as the bars. Before going into an in-depth analysis of our results, we observe that all photons with energies greater than 80 keV have indistinguishable efficiencies - all the way up to ${ }^{137} \mathrm{Cs}$ gamma rays.

Interestingly, none of the samples are either systematically above or below the average (see Table S1), which confirms that there was no systematic gradient in X-ray irradiation geometry, since each sample was always in the same position on the holder, for all irradiations. However, sample 031301 seems to show a lower efficiency than other samples at low energies ( $E<100 \mathrm{keV}$ ); the low sensitivity of this sample to low energy $\mathrm{x}$-rays was also observed [24]. Conversely, for higher photon energies, the response of this sample seems to be greater than average. At this stage, we cannot provide an explanation for this divergent behaviour. The average response of the samples can be used to derive the following general conclusions about quartz OSL.

(i) There is a systematic increase in the average efficiency from 8 to $80 \mathrm{keV} x$-rays. The two lowest energy points on the figure are not statistically different from each other, giving the minimum efficiency of $6 \%$ for $\sim 12 \mathrm{keV}$ photons compared to the ${ }^{137} \mathrm{Cs}$ gamma irradiation.

(ii) The average efficiency of x-rays from 100 to $209 \mathrm{keV}$ (4 different points in Fig. 4a; see also Table 4) is systematically higher than that of gamma. However, within uncertainties all these efficiency values are consistent with unity, so we cannot conclude at this stage whether or not photons in the energy range between 100 and $209 \mathrm{keV}$ are similar to or more effective than ${ }^{137} \mathrm{Cs}$ gamma rays in producing OSL.

(iii) The Relative Standard Deviation (RSD) of equivalent doses measured from the 7 samples is much larger for $x$-ray irradiations than for ${ }^{137}$ Cs gamma irradiations (Fig. 4b). There is $\sim 60 \%$ RSD for the lowest energy of $8-12 \mathrm{keV}$; it subsequently varies between 10 and $4 \%$ from 12 to $250 \mathrm{keV}$, with a weak tendency for a decrease in the RSD with x-ray energy. In case of ${ }^{137} \mathrm{Cs}$, the RSD is only $1 \%$ (dashed line in Fig. $4 \mathrm{~b}$ ). 


\section{ACCEPTED MANUSCRIPT}

The relative increase in efficiency with energy up to $\sim 80 \mathrm{keV} \mathrm{X-rays} \mathrm{can} \mathrm{be} \mathrm{attributed} \mathrm{to} \mathrm{micro-}$ dosimetric effect (high ionisation density) reported in [4]. The high RSD in efficiency for 8 and 12 $\mathrm{keV}$ cannot be attributed to counting statistics; the uncertainty on the estimates of efficiency for an individual sample is typically less than $5 \%$. We consider that a RSD of $\sim 5 \%$ may be attributed to spatial and temporal variations in $\mathrm{x}$-ray flux as well as to combined measurement uncertainties; the remaining RSD (Fig. 4b, Table S1) is then possibly related to different microdosimetric effects in low energy $\mathrm{x}$-ray interactions compared to the ${ }^{90} \mathrm{Sr} /{ }^{90} \mathrm{Y}$ beta irradiation. These differences can for example arise from different dose saturation characteristics in different samples (note that high ionisation density leads to local saturation effects). In terms of practical implications, this dispersion in OSL response to low energy $x$-ray irradiations from one sample to another confirms that a $50 \mathrm{kV}$ x-ray tube without significant shielding is not a good surrogate for

${ }^{137}$ Cs irradiations (and thus true doses), as also concluded by [24]. Our results explains why placing an Al filter in front of the $x$-ray source to harden the spectra, as reported by [24], leads to a reduction in the dispersion in equivalent doses delivered by $\mathrm{x}$-rays. Nonetheless, in the light of our results any $\mathrm{x}$-ray source with photon energies below $\sim 80 \mathrm{keV}$ will give rise to microdosimetric effects and should thus be avoided.

\subsection{K-feldspar $I R_{50}$ and $p I R I R_{290}$ results}

Figs. $5 a$ and $5 b$ show the efficiency of $x$-rays as a function of the photon mean energy for feldspar $I R_{50}$, and pIRIR 290 respectively. It was suggested that the $I R_{50}$ results from the pIRIR 290 protocol may not be reliable because the sensitivity changes occurring after natural signal readout may not be monitored appropriately [32]. However, the results of [32] do not strictly apply here, since our feldspar samples were sensitised by heating before the $x$-ray irradiations. The doserecovery ratios obtained with the $\mathbb{I R}_{50}$ signal were within $5 \%$ of unity, although not consistent with unity at two standard errors; these results suggest that any changes of the order of $5 \%$ or less in the $\mathrm{IR}_{50}$ data should be treated with caution.

The variation in efficiency as a function of x-ray energy for the two samples studied here shares some common behaviour with the quartz OSL. The behaviour of the $I R_{50}$ and pIRIR $R_{290}$ signals is rather similar, which is not surprising given our understanding that these two signals likely arise from the same electron trap $[33,34]$. Some general conclusions can be drawn from these data:

(i) The low energy $x$-rays $(<50 \mathrm{keV})$ are less efficient than ${ }^{137} \mathrm{Cs}$ gamma rays in producing $\mathrm{IR}_{50}$ or pIRIR 290 , relative to ${ }^{137} \mathrm{Cs}$. 


\section{ACCEPTED MANUSCRIPT}

(ii) Unlike quartz which reaches a constant value of $\sim 1$ at $83 \mathrm{keV}$, both $\mathbb{R}_{50}$ and $\mathrm{pIRI} \mathrm{R}_{290}$ show a peakshaped behaviour above $65 \mathrm{keV}$. At $65 \mathrm{keV}$ the average efficiency is close to 1 and then it reaches a peak value of $\sim 1.6$ at $118 \mathrm{keV}$ and then decreases gradually back to about $\sim 1.1$ at $250 \mathrm{keV}$.

(iii) There is a discontinuity in the $I R_{50}$ efficiency curve at $E=48 \mathrm{keV}$ but not in the pIRIR $R_{290}$ data. This is intriguing since both these signals as are measured on the same aliquots. One can be confident that the efficiency dip is not related to irradiation, since both pIRIR ${ }_{290}$ signal (measured on the same aliquot as IRSL) and quartz OSL (quartz was irradiated together with feldspar) do not show this dip. This is most likely related to a physical process in feldspar $\mid \mathbb{R}_{50}$.

(iv) A discontinuity is present in both $\mathrm{IR}_{50}$ and $\mathrm{pIRIR} \mathrm{R}_{290}$ data at $\mathrm{E}=12 \mathrm{keV}$ ( $8 \%$ and $7 \%$ relative efficiency for pIRIR 290 and $I R_{50}$ signals, respectively). This discontinuity also seems to be present in quartz OSL ( $\mathrm{N}-15$ series); however, in contrast to the quartz data the difference in efficiency between the $\mathrm{N}-10$ $(\mathrm{E}=8 \mathrm{keV})$, and $\mathrm{N}-15(\mathrm{E}=12 \mathrm{keV})$ series is statistically significant. Further investigations are required to understand whether the discontinuity at $12 \mathrm{keV}$ is physical or due to some unrecognised source of error. For example, if we have slightly overestimated the thickness of the lid of the container, the dose determined by GEANT4 simulations would be underestimated giving rise to an over-estimate of efficiency; this effect could be significant for the lowest energy because of the small mean free path. However, equally there may be selective excitation of core levels at specific energies (e.g. at $8 \mathrm{keV})$, leading to higher-than-average ionisation density; this may lead to a larger reduction in the efficiency than that expected from the average trend.

Since the shape of feldspar IRSL decay curve is sensitive to the trapped hole density and hence to dose $[35,36]$, we further explored the relationship between IRSL decay and x-ray energy. If $x$-rays at low energies result in high ionisation density (high localised dose), then it should be reflected in the IRSL decay shape. The signal decay should be faster for low energy photons, because of an increase in recombination efficiencies of trapped electrons with nearby trapped holes [35, 36]. We examined the IRSL shine down curves (Fig. 6) using the recent model describing the kinetics of infrared luminescence from feldspar [36]. Since both samples show similar behaviours in terms of luminescence efficiency (Figs 5a and 5b), we focused on only one of them (sample 963602). To optimise the signal to noise ratio, for each energy we created an IRSL decay curve from a 'superlarge' aliquot by summing the signals measured on 5 aliquots. The background, estimated with the last 20s of stimulation, was subtracted from the IRSL signals. Then, based on Eq. (15) in [36], all IRSL shine-down curves were simultaneously fitted with the function:

$$
L(t)=\frac{n_{0}{ }^{\prime}}{\tau_{0}+z t}\left(\operatorname { L n } ( A ( \tau _ { 0 } + z t ) ) ^ { 2 } \operatorname { e x p } \left(-\rho^{\prime}\left(\operatorname{Ln}\left(A\left(\tau_{0}+z t\right)^{3}\right)\right.\right.\right.
$$




\section{ACCEPTED MANUSCRIPT}

Here $L$ is the time $(t)$ dependent IRSL signal intensity, $n_{0}{ }^{\prime}$ is a dose-dependent parameter, $z$ is a constant shared by all curves set to 1.8 (based on [37]), $\tau_{0}$ defines the lifetime at the fading front at the beginning of the measurement and $A$ is the optical excitation probability $\left(s^{-1}\right)$ scaled with the ratio of the attempt-to-tunnel frequency factor and the lattice vibrational frequency factor. The parameter $\rho^{\prime}$ is the dimensionless density of the occupied hole sites [36]; this parameter should decrease with increasing photon energy because of the microdosimetric effects. The parameter $n_{0}{ }^{\prime}$ is proportional to $\rho^{\prime}$ and the trapped electron concentration at time $t=0$; this parameter was allowed to vary from one curve to the other since it is expected that the local density of trapping states will vary with X-ray energy. $\tau_{0}$ was shared by all curves, since preheat should be the dominant factor in moving the fading front. $A$ was shared by all curve since the excitation light flux was constant for all the IRSL measurements.

Figures $7 \mathrm{a}$ and $7 \mathrm{~b}$ show the variation in $\rho^{\prime}$ as a function of $\mathrm{x}$-ray energy for the natural, first test dose and first regenerative dose signals (the latter two signals resulting from laboratory beta dose). The curves for $I_{50}$ and pIRIR ${ }_{290}$ signals show a broadly similar behaviour. Although there is some scatter, there is a tendency for $\rho^{\prime}$ to decrease with increasing $x$-ray energy. Interestingly, the regenerative and test dose signals keep the memory of the luminescence decay shape observed for the initial $\mathrm{x}$-ray irradiations; for a given $\mathrm{x}$-ray energy, the values of $\rho^{\prime}$ estimated using the signals produced by $x$-ray irradiation are indistinguishable from those estimated for the subsequent test dose or the first regeneration dose. Thus, the observed trend of decreasing $\rho^{\prime}$ with increasing photon energy is visible even for the test dose or regeneration dose (note that all test doses or regeneration doses are identical, irrespective of the prior $x$-ray energy). This observation suggests that the density of occupied hole sites during the SAR protocol is governed by the initial $x$-ray irradiations.

\section{Discussion}

We first discuss the mechanism governing the energy response of the optically stimulated luminescence signals in quartz and feldspar, and then discuss its implication for dating.

\subsection{Relative luminescence efficiency as a function of $x$-ray energy}

Our data suggests that for both quartz OSL and feldspar IRSL signals, low energy photons $(\sim<60 \mathrm{keV})$ are less efficient in inducing luminescence signals than ${ }^{137} \mathrm{Cs}$ gamma rays. For quartz, there is a systematic increase in relative efficiency (except for a dip at $12 \mathrm{keV}$ ) with energy reaching a constant value of $\sim 1$ at $\sim 83 \mathrm{keV}$. Our data confirm the model that low energy photons lead to local saturation of the electron/hole traps in the crystals due to high ionisation density, thus decreasing 


\section{ACCEPTED MANUSCRIPT}

the overall trapping efficiency per given dose [4]; this effect is similar to what is observed for OSL induced by alpha irradiation. For example, the lowest efficiency values of $0.06-0.10$ for photons with $E<15 \mathrm{keV}$ are about twice the size of the commonly reported alpha efficiency values for quartz OSL (from 0.02 to 0.05 [3]). While the question of low efficiency for low energy $x$-rays has already been discussed in detail [4], the present data for the first time quantify the extent of this effect in different quartz samples. Furthermore, it is shown that there exists a sample-to-sample variability in response to ionising photons below $80 \mathrm{keV}$; this agrees well with our interpretation above that microdosimetric effects are important below this energy since intra sample variability in saturation dose will affect its efficiency (see detailed discussion in [4]). Both the mean efficiency and its spread across samples suggest that micro-dosimetric effects become insignificant at and above $\sim 83 \mathrm{keV}$, where the quartz OSL response to $\mathrm{X}$-rays becomes similar to the high energy ${ }^{137} \mathrm{Cs}$ irradiation. Thus, for the typical distribution of trapping states in quartz, the microscopic dose distribution for $\geq 80 \mathrm{keV}$ photons can be considered to be uniform.

The efficiency response of feldspar $\mathbb{I R}_{50}$ and $\mathrm{pIRI} \mathbf{R}_{290}$ is more complex. The efficiency of $\mathbf{I}_{50}$ and pIRIR 290 continues to increase without reaching a plateau like quartz; it overshoots $\sim 1$ at $65 \mathrm{keV}$, reaches a peak value of about $\sim 1.6$ at $118 \mathrm{keV}$, and then gradually falls off towards $\sim 1$ for higher $\mathrm{x}$ ray energies. The efficiency for the $<15 \mathrm{keV}$ photons $\left(0.13\right.$ and 0.08 for $\mathrm{IR}_{50}$, respectively; 0.15 and 0.08 for pIRIR $\left.{ }_{290}\right)$ are of the same order as the a values reported for the alpha particles $\left(\sim 0.08\right.$ for $I R_{50}$, e.g. [38]; 0.11 for pIRIR 225 measured on polymineral fine grains, e.g. [39]). Two aspects need to be considered while understanding the micro-dosimetric effect in feldspar compared to quartz. Firstly, the trapping cross-section (partly reflected in the saturation coefficients $\left(D_{0}\right)$ of the beta dose response curve) in feldspar is typically an order of magnitude higher than in quartz compared to feldspar. Secondly, the density of trapping states in feldspar is orders of magnitude higher than quartz leading to localised recombination effects such as tunnelling.

Thus, an increase in ionisation density will lead on the one hand to localised trap saturation, similar to quartz, but also to a relatively higher number density of holes around the electron trap, thus enhancing the probability of recombination (see detailed discussion in [33]). These two effects (trap saturation on the one hand, increased recombination probability on the other) compete with each other giving rise to a peak shaped behaviour. To illustrate this principle, we fitted the efficiency curve of quartz OSL as a function of x-ray energy (Fig. 4a) with a single saturating exponential function of the form $\xi(E)=\xi_{\infty}\left(1-e^{a E}\right)$, where $a$ is the exponential constant and $E$ is the x-ray energy. Similarly, we also fitted the evolution of $\rho^{\prime}$ as a function of x-ray energy (Fig. 7) with a decaying exponential $\rho^{\prime}(E)=\rho_{0}^{\prime} e^{-b E}$, where $b$ is the exponential constant. The dimensionless hole density is used as a proxy for recombination efficiency [36]. Note that we do not imply here that 


\section{ACCEPTED MANUSCRIPT}

these two quantities evolve exponentially; the purpose here is only to obtain a smooth description of the data. Thus, the choice of exponential functions is entirely arbitrary, and with no physical implication. We then used a product of these two functions to describe the $\mathbb{R}_{50}$ and $p|R| R_{290}$ efficiency data; in doing so, we implicitly hypothesise that feldspar undergoes a micro-dosimetric saturation effect similar to quartz, but the measured luminescence is a function of localised hole density.

The product of the two functions is plotted as the dashed curves in Figs. 5a and 5b. While the general form of the function was maintained, the fitting parameters were adjusted slightly to account for differences in quartz and feldspar in order to obtain a reasonable description of the $\mathbb{I}_{50}$ and $\mathrm{pIRIR}_{290}$ efficiency data. In particular, for the pIRIR 290 efficiency curve, the parameter $a\left(\mathrm{keV}^{-1}\right)$, which characterises the dynamic range of the saturation effect, is somewhat larger than that obtained from the quartz OSL dataset; this is compatible with the fact that the dose saturation coefficient in feldspar is larger than in quartz, thus the decrease in efficiency with x-ray energy occurs more gradually. The good agreement between the dashed curve and the experimental data validates our hypothesis, thus suggesting that the feldspar efficiency response is sensitive to the saturation of electron traps (as in the case of quartz), as well as to a change in the density of trapped holes. However, this model suggests that the value of $\rho^{\prime}$ for the ${ }^{137} \mathrm{Cs}$ irradiation should be lower than that for high energy $x$-ray irradiations, which would explain why 100-250 keV photons are more efficient than ${ }^{137} \mathrm{Cs}$ rays. But contrary to our expectation the value of $\rho^{\prime}$ for ${ }^{137} \mathrm{Cs}$ irradiations is similar to low energy $\mathrm{x}$-rays. This inconsistency suggests that there some other unknown factor is involved, which needs further investigation.

Looking at Fig. 5a, the dip in the $I R_{50}$ efficiency at $48 \mathrm{keV}$ and the lack of it in the pIRIR $R_{290}$ efficiency curve (Fig. 5b) is also interesting. Looking closely at Fig. 7a showing the evolution of $\rho^{\prime}$ as a function of x-ray energy, one can see that the memory effect described above (section 5) does not seem to apply to the $48 \mathrm{keV}(\mathrm{N} 60)$ irradiation for $\mathrm{IR}_{50}$ : unlike for all other points in the figure, the value of the $\rho^{\prime}$ parameter is significantly higher for the first test dose signal than for the 'natural' ( $x$ ray) signal. Since the efficiency of $I R_{50}$ is based on the test-dose normalised luminescence intensity, it is possible that the dip in the $\mathbb{I R}_{50}$ response to $\mathrm{x}$-ray energy is explained by an anomalously low recombination efficiency at this $\mathrm{x}$-ray energy. It is possible that this is related to some resonance interaction, leading to closely spaced filling of electrons and holes; thus, the dip is only present in $I_{50}$ and not in pIRIR 290 . Here again, more investigation would be required to understand the phenomenon. 


\section{ACCEPTED MANUSCRIPT}

Finally, we also compare the shapes of the $\mathbb{I R}_{50}$ decay curves for three different irradiation conditions: ${ }^{90} \mathrm{Sr} /{ }^{90} \mathrm{Y}$ (from beta dose recovery experiments), $250 \mathrm{keV}$ (N300 $\mathrm{x}$-ray source) photons, and 1.17 and $1.33 \mathrm{MeV}\left({ }^{137} \mathrm{Cs}\right)$ photons. The comparison is shown in Fig. 8. The beta induced luminescence signal decays faster than the signal induced by ${ }^{137} \mathrm{Cs}$ gamma rays, which in turn decays faster than the signal induced by $250 \mathrm{keV}$ photons (the latter is reflected in the higher $\rho^{\prime}$ value for

${ }^{137} \mathrm{Cs}$ compared to $250 \mathrm{keV}$ photons, $c f$. Fig. 8). These differences in $\mathrm{IR}_{50}$ decay rates suggest that ${ }^{137} \mathrm{Cs}$ irradiations may perhaps have different micro-dosimetric effects compared to ${ }^{90} \mathrm{Sr} /{ }^{90} \mathrm{Y}$; these data raise an issue regarding the possibility to use feldspar for direct calibration of the beta source. Perhaps more importantly, just like we did not expect gamma irradiations to lead to faster decay of IRSL signals, we also cannot explain why beta-induced signals decay faster than signals induced by $x$ rays (N-300). Thus, although our model for feldspar describes well the observed variation in luminescence efficiency as a function of x-ray energy, it does not explain the inter-comparison of $\rho^{\prime}$ across X-ray, beta and gamma irradiations.

\subsection{Consequences for luminescence dating}

A first technical implication of our study is that in order to use $\mathrm{x}$-ray source as a replacement for beta source for quartz OSL dating, the mean x-ray energy should be greater than $80 \mathrm{keV}$; otherwise, the sample-to-sample variability in luminescence efficiency of quartz is significant and will require a sample-specific calibration. In case of feldspar, we do not have enough samples to address this issue, however, it appears that micro-dosimetric effects exist up to much higher $\mathrm{x}$-ray energies than quartz. Our limited dataset suggests that an $\mathrm{x}$-ray tube delivering a mean energy of $80 \mathrm{keV}$ could potentially replace the beta source.

More fundamentally, our results suggest that all photons and electrons, in the energy range of natural and laboratory irradiations, are not equivalent in terms of luminescence efficiency; this invalidates one of the key assumptions of luminescence dating. For quartz OSL dating, one may deduce from Fig. 4a that beta dose rate from the Thorium series (average primary electron energy $\bar{E}=186 \mathrm{keV})$ should be less efficient than that from the Uranium series $(\bar{E}=246 \mathrm{keV})$, which in turn should be less efficient than that from Potassium $(\bar{E}=509 \mathrm{keV})$ in producing luminescence output per unit dose (the mean electron energies were deduced from the beta spectra of [40]). This effect should be less pronounced for gamma dose rates; the average primary photon energies are $614 \mathrm{keV}$ (Th), $537 \mathrm{keV}$ ( $\mathrm{U}$ - these average energies were calculated from the gamma spectra of [41]) and 1461 $\mathrm{keV}(\mathrm{K})$, all well above the energy at which local saturation effects appear. Our results suggest that beta (and possibly gamma) conversion factors, should be revisited to consider possible microdosimetric effects on luminescence behaviour. One may develop radionuclide-dependent 


\section{ACCEPTED MANUSCRIPT}

"effective dose rate" conversion factors in a similar manner to those used presently for the alpha dose rates. Furthermore, the luminescence efficiency curves for K-feldspar IRSL signals differ from that of quartz OSL. While further understanding of the processes in feldspar is necessary, it appears that the effective dose rate conversion factors for feldspar will be different to those for quartz.

This study also raises the question of calibration of the laboratory dose rate. The laboratory source of ionising radiation should reflect the energy spectrum to which the minerals were exposed during burial. The measured dose rate in the laboratory is essentially an effective dose rate based on matching of luminescence response; thus, it includes the luminescence efficiency of different radiation sources that are used in the calibration procedure. It appears that the procedure for measuring dose rate for quartz should be fine since the mean energy of ${ }^{90} \mathrm{Sr} /{ }^{90} \mathrm{Y}$ is $\sim 560 \mathrm{keV}$, whereas

${ }^{137} \mathrm{Cs}$ emits two gamma rays at 1.17 and $1.33 \mathrm{MeV}$, respectively. Adapting the same procedure may lead to inaccurate result for feldspar, since the luminescence efficiency is a non-first order, competition effect, which is affected both by trapping and recombination efficiency. Thus, feldspar is not a suitable material for dose-rate calibration; the dose rate to feldspar should therefore be derived based on quartz (as is done commonly). Furthermore, the efficiency of the laboratory beta source in producing luminescence needs to be compared with the natural spectrum, in order to quantify the correction factors; this is deemed especially important for feldspar.

Finally, this study also has consequences on equivalent dose variability of quartz single grain OSL. In cases where the infinite matrix assumption is not verified (which in practice is very common see e.g., $[42,40,43])$, the spatial variability of effective luminescence dose rates should be greater than the spatial variability of absolute dose rates. At the scale of a single grain, let us consider two quartz grains, $\mathrm{A}$ and $\mathrm{B}$, such that $\mathrm{A}$ is closer to a beta hotspot (e.g., a K-feldspar grain) than $\mathrm{B}$. Not only will $A$ and $B$ experience different dose rates depending on their distance to the hotspot, but also different electron-energy spectra. If $B$ is so far from the source that it only receives low energy electrons nearing the end of their track, the effective dose rate will be much lower than the true dose rate. In other words, the luminescence efficiency effect explored in this paper will further amplify the effect of grain-to-grain variability in dose rate on the net OSL signal [43], depending on the energy spectrum of the ionising electrons.

\section{Conclusion}

We presented the first measurement of the energy response of the luminescence signals from quartz $(\mathrm{OSL})$ and $\mathrm{K}$-feldspar ( $\left(\mathrm{R}_{50}\right.$ and pIRIR 290 ) to photon irradiation in the range of 8 to $250 \mathrm{keV}$. 


\section{ACCEPTED MANUSCRIPT}

We infer that micro-dosimetric effects at low photon energies ( $<80 \mathrm{keV}$ for quartz and $<120 \mathrm{keV}$ for K-feldspar) lead to a systematic decrease in the luminescence efficiency (OSL or IRSL intensity per unit dose) with a decrease in photon energy; at the smallest energies the efficiencies are comparable with those from natural alpha irradiations. These micro-dosimetric effects also lead to significant sample to sample variation in the OSL response of quartz.

The energy response of K-feldspar is more complex than quartz. The $I R_{50}$ and pIRIR $R_{290}$ responses appear to result from a competition between trap saturation (decreased trapping efficiency) along the tracks of low energy electrons (similar to quartz), and an increased recombination efficiency due to a greater number density of holes available for recombination. This gives rise to a peak-shaped energy response. Further investigations are required to understand this fully.

Our results have several implications for dating applications. Firstly, specific dose-rate conversion factors for $\mathrm{K}, \mathrm{U}$ and Th should take into account the reduced efficiency of low energy photons and electrons in producing luminescence. These factors are likely to be different for quartz and feldspar. Secondly, a comparison of the energy spectra in the natural environment and that for the laboratory irradiation source should be revisited in the light of our results. Finally, if a beta source should be replaced by an $\mathrm{x}$-ray tube for laboratory irradiations, then it should at least deliver a mean energy of $80 \mathrm{keV}$.

\section{Acknowledgements}

GG acknowledges H.C. Ørsted postdoctoral fellowship (2011-2013) from the Technical University of Denmark to undertake this research.

\section{References:}

[1] Aitken, M. J., 1985. Thermoluminescence dating. Academic Press, London.

[2] Huntley, D.J., 2001. Note: some notes on language. Ancient TL 19, 27-28.

[3] Mauz, B., Packman, S. and Lang, A., 2006. The alpha efficiency of silt-sized quartz: New data obtained by single and multiple aliquot protocols. Ancient TL 24, 47-52.

[4] Jain, M., Bøtter-Jensen, L., Thomsen, K.J., 2007. High local ionization density effects in x-ray excitations deduced from optical stimulation of trapped charge in Al2O3:C. Journal of Physics of Condensed Matter 19, 116-201.

[5] Davis, S. D., Ross, C. K., Mobit, P. N., Van Der Zwan, L., Chase, W. J., Shortt, K. R., 2003. The response of LiF thermoluminescence dosemeters to photon beams in the energy range from $30 \mathrm{kV} \mathrm{x}$ rays to 60 Co gamma rays. Radiation Protection Dosimetry 106, 33-43. 


\section{ACCEPTED MANUSCRIPT}

[6] Nunn, A. A., Davis, S. D., Micka, J. A., Dewerd, L. A., 2008. LiF:Mg, Ti TLD response as a function of photon energy for moderately filtered x-ray spectra in the range of 20-250 kVp relative to Co-60. Medical Physics 35, 1859-1869.

[7] Sommer, M., Freudenberg, R., Henniger, J., 2007. New aspects of a BeO-based optically stimulated luminescence dosimeter. Radiation Measurements 42, 617-620.

[8] Gasparian, P.B.R., Vanhavere, F., Yukihara, E.G., 2012. Evaluating the influence of experimental conditions on the photon energy response of Al2O3:C optically stimulated luminescence detectors, Radiation Measurements 47, 243-249.

[9] Malthez, A. L., Freitas, M. B., Yoshimura, E. M., \& Button, V. L., 2014. Experimental photon energy response of different dosimetric materials for a dual detector system combining thermoluminescence and optically stimulated luminescence. Radiation Measurements 71, 133-138.

[10] Bøtter-Jensen, L., Andersen, C.E., Duller, G.A.T., Murray, A.S., 2003. Developments in radiation, stimulation and observation facilities in luminescence measurements. Radiation Measurements 37, 535-541.

[11] Bøtter-Jensen, L., Thomsen, K.J., Jain, M., 2010. Review of optically stimulated luminescence (OSL) instrumental developments for retrospective dosimetry. Radiation Measurements 45, 253-257.

[12] Murray, A. S., Wintle, A. G., 2000. Luminescence dating of quartz using an improved singlealiquot regenerative-dose protocol. Radiation Measurements 32, 57-73.

[13] Madsen, A.T., Murray, A.S., Andersen, T.J., 2007. Optical dating of dune ridges on Rømø, a Barrier Island in the Wadden Sea, Denmark. Journal of Coastal Research 23, 1259-1269.

[14] Buylaert, J.-P., Jain, M., Murray, A.S., Thomsen, K.J., Thiel, C., Sohbati, R., 2012. A robust feldspar luminescence dating method for Middle and Late Pleistocene sediments. Boreas 41, 435-451.

[15] Thomsen K.J., Murray A.S., Bøtter-Jensen L., Kinahan J., 2007. Determination of burial dose in incompletely bleached fluvial samples using single grains of quartz. Radiation Measurements 42, 370379.

[16] Murray, A.S., Funder, S., 2003. Optically stimulated luminescence dating of a Danish Eemian coastal marine deposit: a test of accuracy, Quaternary Science Reviews, 22 (10-13), 1177-1183.

[17] Geach, M.R., Thomsen, K.J., Buylaert, J.-P., Murray, A.S., Mather, A.E., Telfer, M.W., Stokes, M., 2015. Single-grain and multi-grain OSL dating of river terrace sediments in the Tabernas Basin, SE Spain. Quaternary Geochronology, 30, 213-218.

[18] Murray, A.S., Buylaert, J.-P., Henriksen, M., Svendsen, J.I., Mangerud, J., 2008. Testing the reliability of quartz OSL ages beyond the Eemian. Radiation Measurements 43, 776-780.

[19] Leopold, M., Voelkel, J., Dethier, D. P., 2008: Near surface geophysics and sediment analysis to precisely date the outburst of glacial Lake Devlin, Front Range Colorado, USA. Geophysical Research Abstracts 10, EGU2008-A-10437.

[20] Sørensen R., Murray A.S., Kaaya A.K., Kilasara M., 2001. Stratigraphy and formation of a Late Pleistocene colluvial apron in Morogoro District, central Tanzania. Palaeoecology of Africa 27, 95116.

[21] Choi, J.H., Murray, A.S, Jain, M., Cheong, C.S., Chang, H.W., 2003. Luminescence dating of wellsorted marine terrace sediments on the southeastern coast of Korea. Quaternary Science Reviews $22,233-247$. 


\section{ACCEPTED MANUSCRIPT}

[22] Jain, M., Murray, A.S., Bøtter-Jensen, L., 2003. Characterisation of blue-light stimulated luminescence components in different quartz samples: implications for dose measurement. Radiation Measurements 37, 441-449.

[23] Jain, C., Murray, A.S., B $\emptyset$ tter-Jensen, L., Wintle, A.G., 2005. A single-aliquot regenerative-dose method based on IR $(1.49 \mathrm{eV})$ bleaching of the fast OSL componentin quartz. Radiation Measurements 39, 309-318.

[24] Thomsen, K.J., Bøtter-Jensen, L., Denby, P.M., Murray, A.S., 2006. Luminescence response to irradiation using mini X-ray generators, Nuclear Instruments and Methods in Physics Research Section B: Beam Interactions with Materials and Atoms 252, 267-275.

[25] Thiel, C., Buylaert, J.P., Murray, A., Terhorst, B., Hofer, I., Tsukamoto, S., Frechen, M., 2011. Luminescence dating of the Stratzing loess profile (Austria) - testing the potential of an elevated temperature post-IR IRSL protocol. Quaternary International 234, 23-31.

[26] Hansen, V., Murray, A.S., Buylaert, J.-P., Yeo, E.-Y., Thomsen, K.J., 2015. A new irradiated quartz for beta source calibration. Radiation Measurements 81, 123-127.

[27] Bos, A.J.J., Wallinga, J., Johns, C., Abellon, R.D., Brouwer, J.C., Schaart, D.R., Murray, A.S., 2006. Accurate calibration of a laboratory beta particle dose-rate for dating purposes. Radiation Measurements 41, 1020-1025.

[28] ISO International Organisation for Standardisation, 1996. $\mathrm{X}$ and gamma reference radiation for calibrating dosemeters and doserate meters and for determining their response as a function of photon energy - Part 1: Radiation characteristics and production methods. ISO 4037-1:1996€.

[29] Agostinelli S., et al. (Geant4 Collaboration) (2003). Geant4 - a simulation toolkit. Nuclear Instruments and Methods A, 506, 250-303.

[30] Allison J., et al. (Geant4 Collaboration) (2006). Geant4 developments and applications. IEEE Transactions on Nuclear Sciences, 53, 270-278.

[31] Salvat, F., Fernandez-Varea, J.M., Acosta, E., Sempau, J., 2011. PENELOPE - A Code System for Monte Carlo Simulation of Electron and Photon Transport. Workshop Proceedings, Issy-lesMoulineaux, France, (November 2001), AEN-NEA.

[32] Kars, R. H., Reimann, T. R., Wallinga, J., 2014. Are feldspar SAR protocols appropriate for post IRIRSL dating? Quaternary Geochronology 22, 126-136.

[33] Jain, M., Ankjærgaard, C., 2011. Towards a non-fading signal in feldspar: insight into charge transport and tunnelling from time-resolved optically stimulated luminescence. Radiation Measurements 46, 292-309.

[34] Andersen, M. T. Jain, M., Tidemand-Lichtenberg, P., 2012. Red-IR stimulated luminescence in Kfeldspar: Single or multiple trap origin? Journal of Applied Physics 112, 043-507.

[35] Jain, M., Guralnik, B., Andersen, M.T., 2012. Stimulated luminescence emission from localized recombination in randomly distributed defects. Journal of Physics of Condensed Matter 24, 385-402.

[36] Jain, M., Sohbati, R., Guralnik, B., Murray, A. S., Kook, M., Lapp, T., Prasad, A.K., Thomsen, K. J., Buylaert, J. P., 2015. Kinetics of infrared stimulated luminescence from feldspars. Radiation Measurements, 81, 242-250.

[37] Pagonis, V., Kitis, G., 2015. Mathematical aspects of ground state tunneling models in luminescence materials. Journal of Luminescence 168, 137-144. 


\section{ACCEPTED MANUSCRIPT}

[38] Rees-Jones, J., Hall, S. J. B., Rink, W. J., 1997. A laboratory inter-comparison of quartz optically stimulated luminescence (OSL) results. Quaternary Science Reviews 16, 275-280.

[39] Kreutzer, S., Schmidt, C., DeWitt, R., and Fuchs, M., 2014. The a-value of polymineral fine grain samples measured with the post-IR IRSL protocol. Radiation Measurements 69, 18-29.

[40] Guérin, G., Mercier, N., Nathan R., Adamiec, G., Lefrais, Y., 2012. On the use of the infinite matrix assumption and associated concepts: a critical review. Radiation Measurements, 47, 778-785.

[41] Guérin, G., Mercier, N., 2011. Determining gamma dose rates by field gamma spectroscopy in sedimentary media: results of Monte Carlo simulations. Radiation Measurements 46, 190-195.

[42] Mayya, Y.S., Morthekai, P., Murari, M.K., Singhvi, A.K., 2006. Towards quantifying beta microdosimetric effects in single-grain quartz dose distribution. Radiation Measurements, 41, 10321039.

[43] Guérin, G., Jain M., Thomsen K. J., Murray A. S., Mercier, N., 2015. Modelling dose rate to single grains of quartz in well-sorted sand samples: the dispersion arising from the presence of potassium feldspars and implications for single grain OSL dating. Quaternary Geochronology 27, 52-65.

\section{Figure Captions}

Fig. 1. Intensity of the first ('natural') 1Gy test dose response as a function of measured to given dose ratios, obtained in $1 \mathrm{~Gy}$ dose experiments. The triangles indicate quartz samples measured with OSL, while the circles correspond to feldspar measured with pIRIR 290 .

Fig. 2. Examples of $x$-rays spectra simulated with GEANT4. These spectra correspond to the narrow $(' N$ ') spectrum series, as specified by the ISO 4037-1 recommendations, and are used for input in the simulations of irradiations of our samples. The bin width was chosen arbitrarily for each distribution to aid visualisation.

Fig. 3. Dose, obtained by Geant4 simulations, delivered to quartz and feldspar grains, as a function of $x$-rays average energy (the emission spectra correspond to the ISO 4037 narrow series; see also Fig. 2 and Table 2).

Fig. 4. (a) Relative efficiency of $x$-rays, as a function of their energy, compared to ${ }^{137} \mathrm{Cs}$ gamma rays, in inducing quartz OSL (see equation (2) in the text). The dashed line indicates the gamma efficiency. (b) Relative standard deviation (RSD) in equivalent doses measured for quartz samples $(n=7)$ after irradiations with $x$-rays of different energies. The RSD obtained with gamma ${ }^{137} \mathrm{Cs}$ irradiations is plotted as the dashed line.

Fig. 5. (a) Relative efficiency of $x$-rays, as a function of their energy, compared to ${ }^{137} \mathrm{Cs}$ gamma rays, in inducing K-feldspar $\mathbb{I R}_{50}$ (from the pIRIR ${ }_{290}$ protocol; see equation (2) in the text). The solid line indicates the gamma ${ }^{137} \mathrm{Cs}$ efficiency. Note the major unconformity for $\mathrm{E}=48 \mathrm{keV}$. (b) Same as (a), but for pIRIR 290 in K-feldspar. See text for the dashed lines.

Fig. 6. 'Natural' (i.e., signal induced by the x-ray irradiations) IRSL signals for different energy x-ray irradiations. The background-subtracted curves were simultaneously fitted by equation (3) to investigate how the dimensionless hole number density $\left(\rho^{\prime}\right)$ varies as a function of $\mathbf{x}$-ray energy.

Fig. 7. Evolution of the dimensionless number density of hole sites $\left(\rho^{\prime}\right)$ as a function of $\mathrm{x}$-ray energy, derived from the analysis of the (a) $I R_{50}$ and (b) $p I R_{290}$ signals. The black squares correspond to the 'natural' signals, the red circles to the test dose signals following the measurement of the natural, and the blue triangles to the signal from the first regeneration beta dose. The lines correspond to the 


\section{ACCEPTED MANUSCRIPT}

value of $\rho^{\prime}$ for Cs gamma irradiations. For all signals, $\rho^{\prime}$ decreases systematically with photon energy; however, the value of $\rho^{\prime}$ for gamma rays is comparable to the value obtained for low energy $\mathrm{x}$-rays and greater than that for high-energy $\mathrm{x}$-rays.

Fig. 8. 'Natural' IRSL $\left(I_{50}\right)$ signals generated by beta ${ }^{90} \mathrm{Sr} /{ }^{90} \mathrm{Y}$, gamma ${ }^{137} \mathrm{Cs}$ and $250 \mathrm{keV}$ photons (N300 series). All three signals appear to be markedly different. 
Figure 1

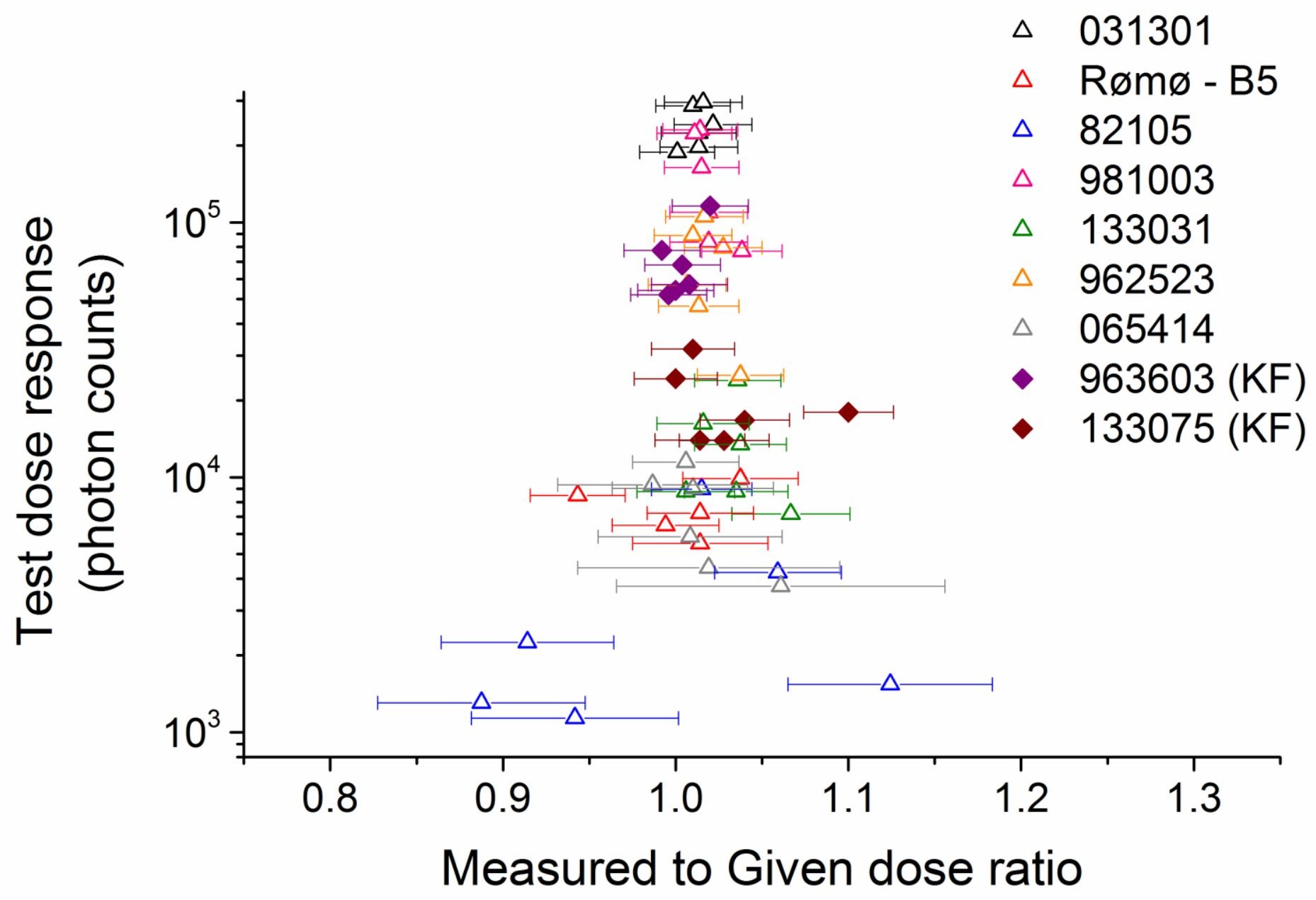


Figure 2
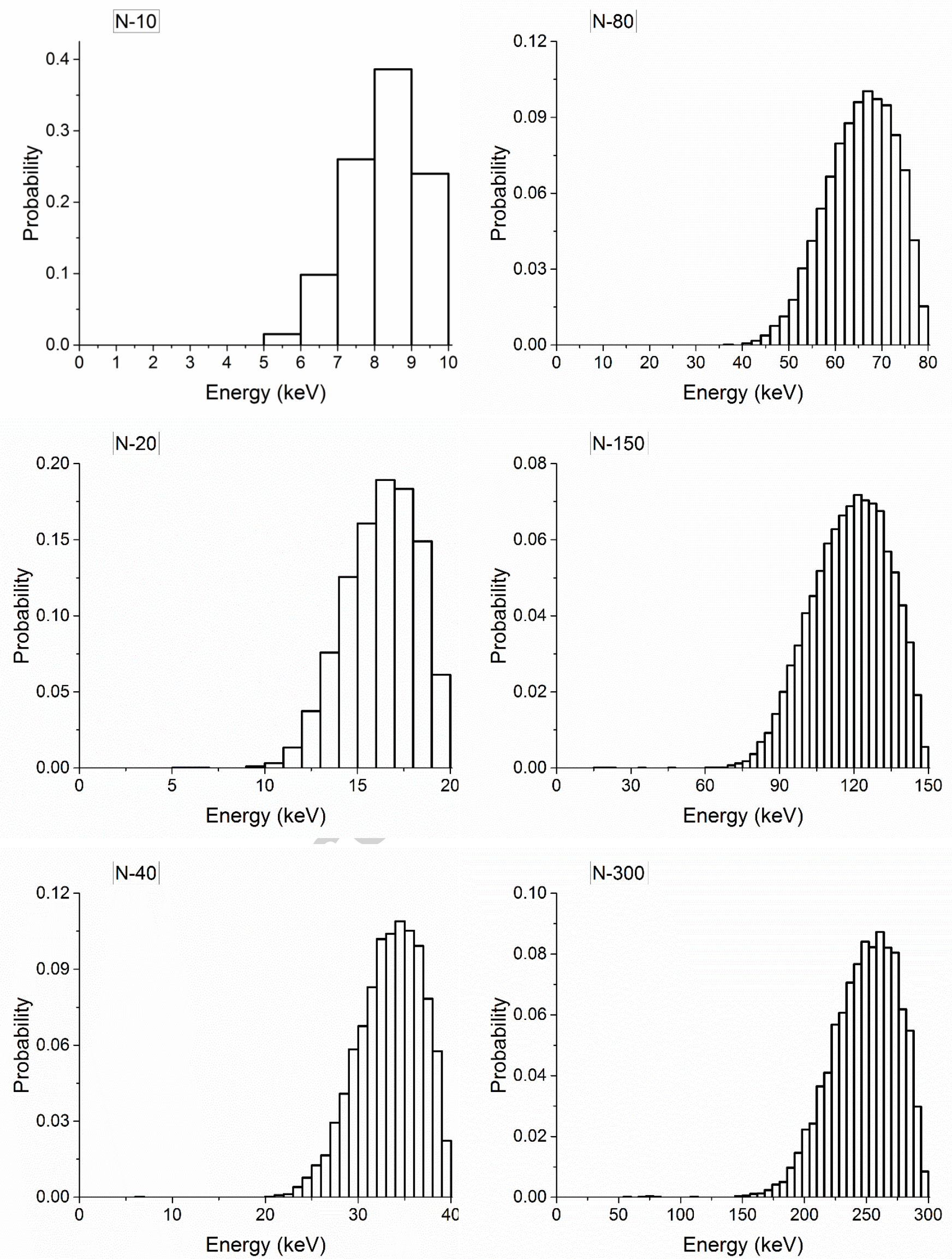
Figure 3

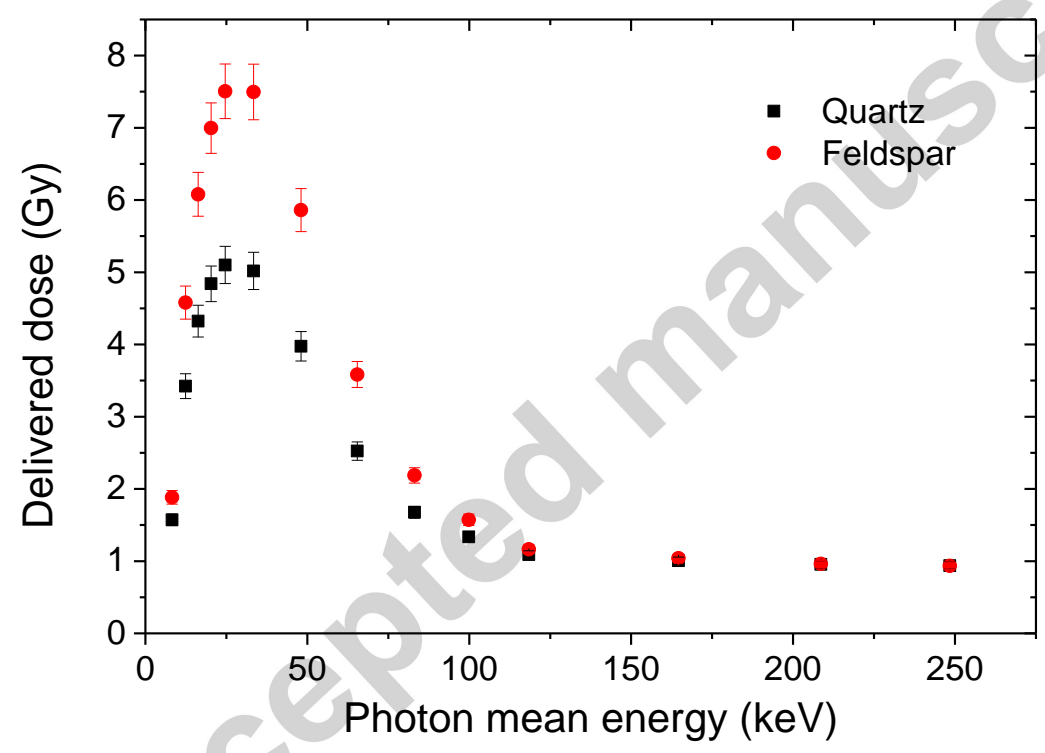


Figure 4
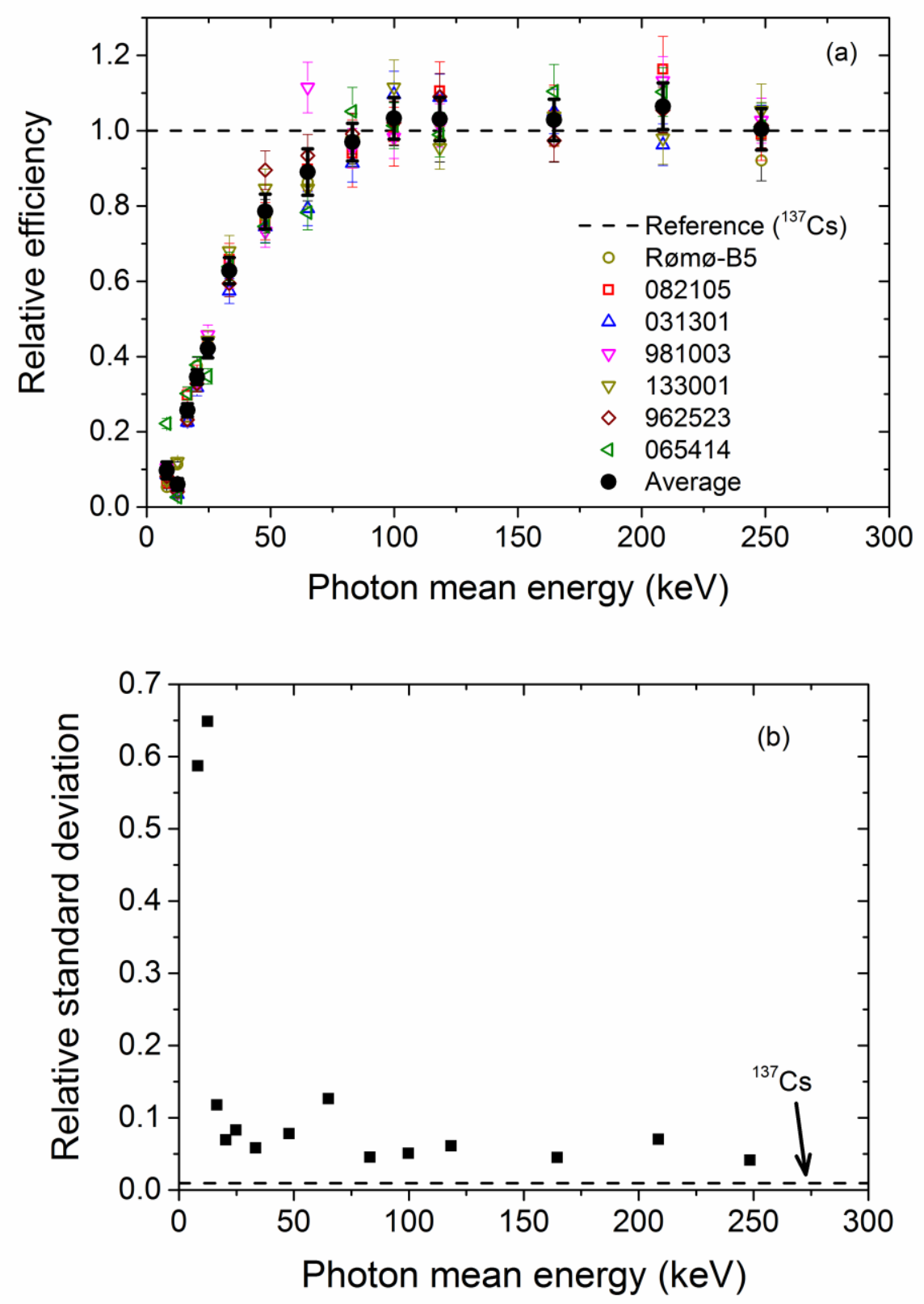
Figure 5
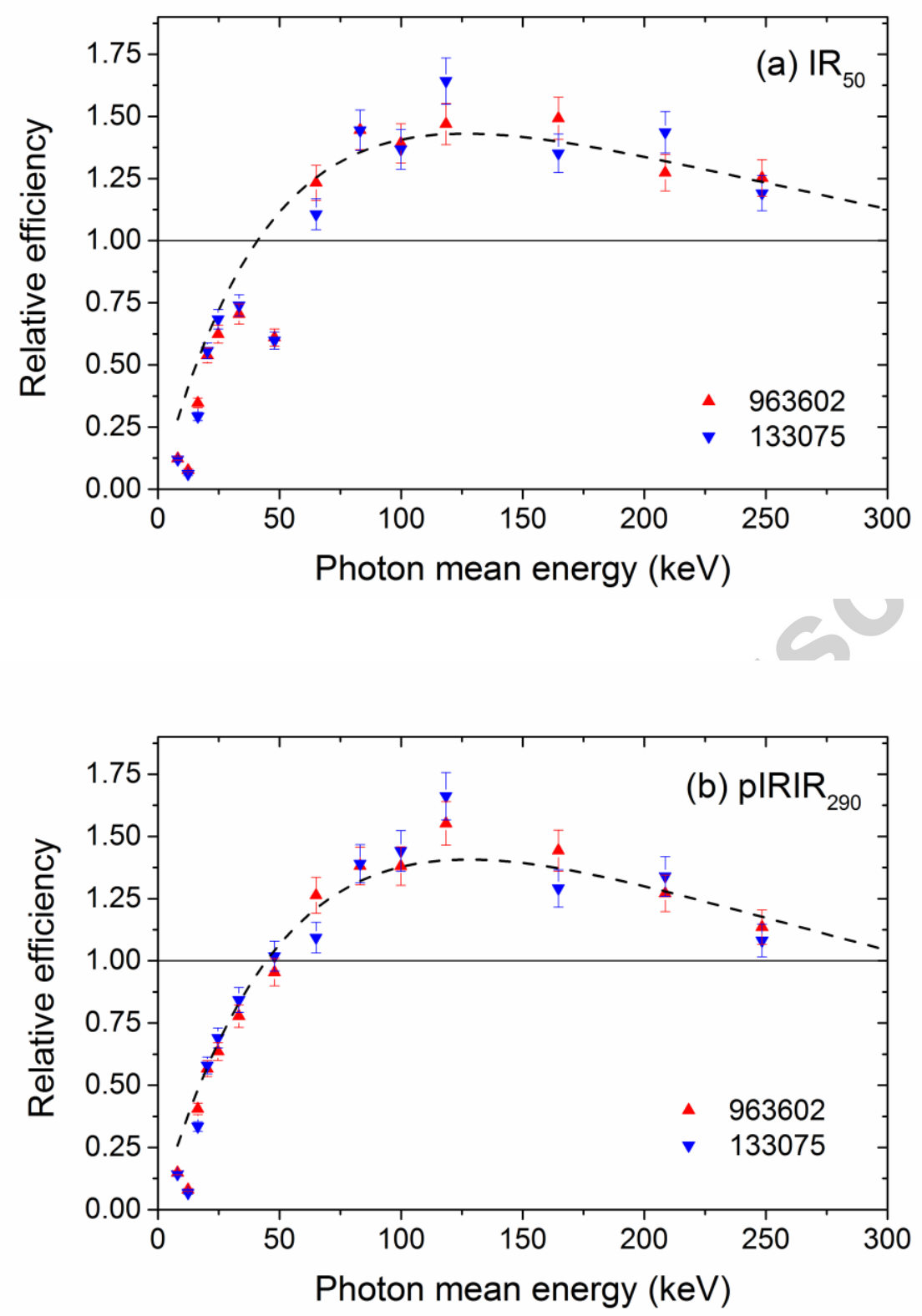


\section{Figure 6}

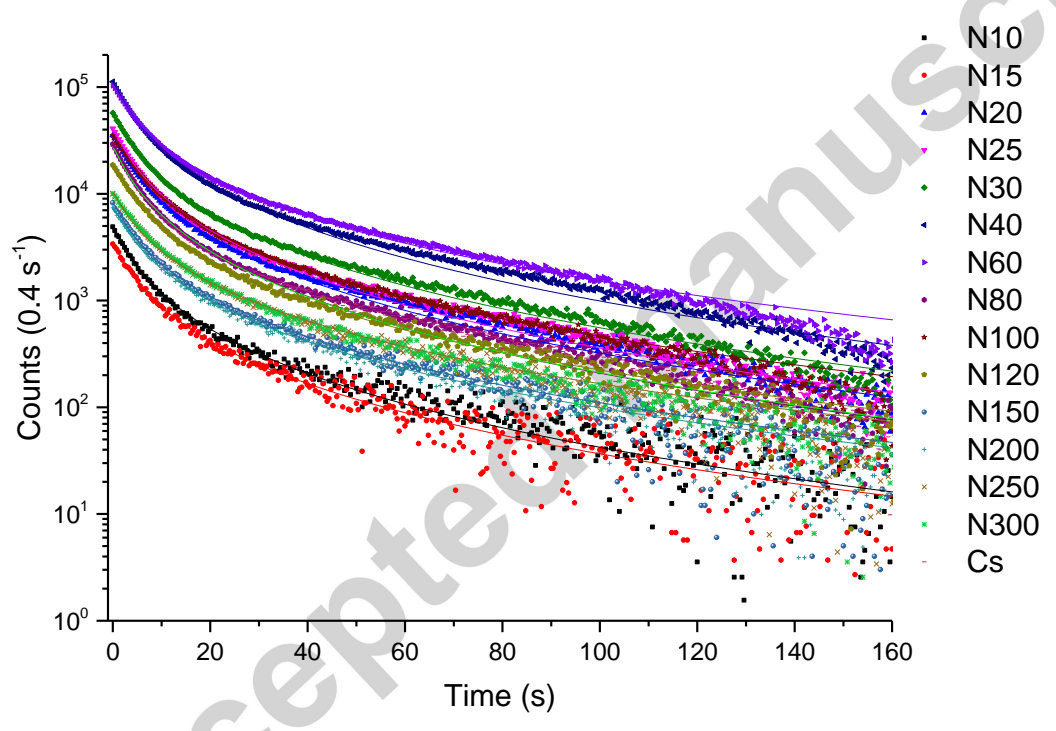


Figure 7
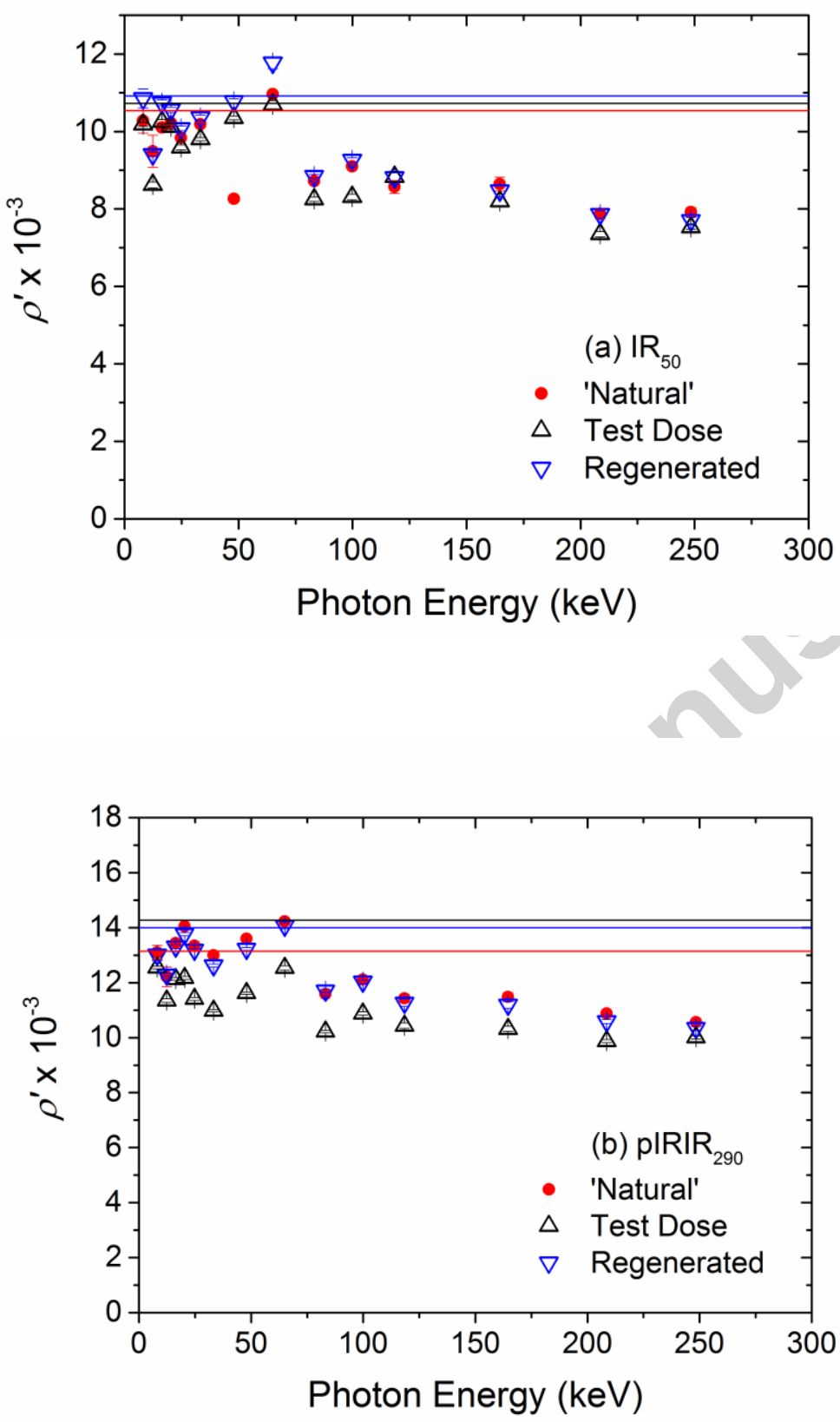
Figure 8

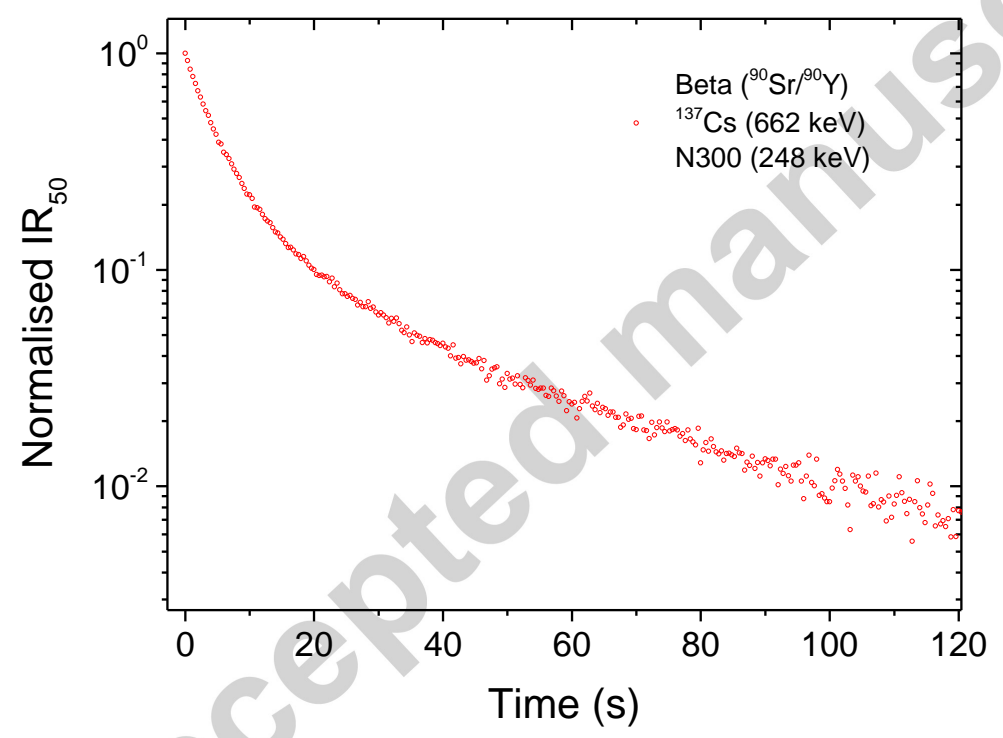


Table 1. List of samples measured in this study. The measured to given dose ratio corresponds to the results of a dose recovery test experiment. The values indicated for K-feldspar samples (963602 and 133075 ) correspond to the pIRIR 290 and $I R_{50}$ (in brackets) protocols, respectively.

\begin{tabular}{|c|c|c|c|c|c|c|}
\hline Provenance & ID & Comment & Mineral & Reference & $\begin{array}{l}\text { Measured } \\
\text { to given } \\
\text { dose ratio }\end{array}$ & $\begin{array}{l}\text { Standard } \\
\text { error }\end{array}$ \\
\hline $\begin{array}{l}\text { Røm } \varnothing \text {, } \\
\text { Denmark }\end{array}$ & B5 & $\begin{array}{l}\text { Unheated } \\
\text { sediment }\end{array}$ & Quartz & $\begin{array}{l}\text { Madsen et al. } \\
\text { (2007) }\end{array}$ & 1.04 & 0.04 \\
\hline $\begin{array}{l}\text { Zwolle, The } \\
\text { Netherlands }\end{array}$ & 082105 & $\begin{array}{l}\text { Unheated } \\
\text { sediment }\end{array}$ & Quartz & $\begin{array}{l}\text { Buylaert et al. } \\
\text { (2012) }\end{array}$ & 0.98 & 0.04 \\
\hline $\begin{array}{l}\text { Linyanti River, } \\
\text { Namibia }\end{array}$ & 031301 & $\begin{array}{l}\text { Ceramics (past } \\
\text { heating) }\end{array}$ & Quartz & $\begin{array}{l}\text { Thomsen et al. } \\
\text { (2007) }\end{array}$ & 1.01 & 0.00 \\
\hline $\begin{array}{l}\text { Gammelmark, } \\
\text { Denmark }\end{array}$ & 981003 & Sensitised & Quartz & $\begin{array}{l}\text { Murray and } \\
\text { Funder (2003) }\end{array}$ & 1.02 & 0.00 \\
\hline $\begin{array}{l}\text { Tabernas, } \\
\text { Spain }\end{array}$ & 133001 & Sensitised & Quartz & $\begin{array}{l}\text { Geach et al. } \\
\text { (2015) }\end{array}$ & 1.03 & 0.01 \\
\hline Seyda, Russia & 962523 & Sensitised & Quartz & $\begin{array}{l}\text { Murray et al. } \\
\text { (2008) }\end{array}$ & 1.02 & 0.00 \\
\hline Colorado, USA & 065414 & Sensitised & Quartz & $\begin{array}{l}\text { Leopold et al. } \\
\text { (2008) }\end{array}$ & 1.02 & 0.01 \\
\hline $\begin{array}{l}\text { Morogoro, } \\
\text { Tanzania }\end{array}$ & 963602 & Sensitised & $\begin{array}{l}\text { K- } \\
\text { feldspar }\end{array}$ & $\begin{array}{l}\text { Sørensen et al. } \\
\text { (2001) }\end{array}$ & $\begin{array}{c}1.00 \\
(1.05)\end{array}$ & $\begin{array}{c}0.00 \\
(0.02)\end{array}$ \\
\hline $\begin{array}{l}\text { Unknown } \\
\text { origin }^{1}\end{array}$ & 133075 & Sensitised & $\begin{array}{l}\text { K- } \\
\text { feldspar }\end{array}$ & $0>-2+2$ & $\begin{array}{c}1.03 \\
(1.03)\end{array}$ & $\begin{array}{c}0.01 \\
(0.01)\end{array}$ \\
\hline
\end{tabular}

${ }^{1}$ : commercial kiln-dried sand.

Table2. Characteristics of the spectra from the narrow-spectrum series: comparison between the ISO recommendations and the results of our GEANT4 simulations. The good agreement between the two data sets indicates that our spectra are representative of the ISO specifications and thus can be used for the irradiation simulations (see text for details).

\begin{tabular}{l|c|c|c|c} 
& \multicolumn{2}{|c|}{ ISO specifications } & \multicolumn{2}{c}{ GeANT4 simulations } \\
Spectrum & Mean Energy (keV) & Dispersion (\%) & Mean Energy (keV) & Dispersion (\%) \\
\hline N-10 & 8 & 29 & 8 & 32 \\
N-15 & 12 & 33 & 12 & 32 \\
N-20 & 16 & 34 & 16 & 33 \\
N-25 & 20 & 33 & 20 & 32 \\
N-30 & 24 & 32 & 25 & 31 \\
N-40 & 33 & 30 & 33 & 28 \\
N-60 & 48 & 36 & 48 & 34 \\
N-80 & 65 & 32 & 65 & 30 \\
N-100 & 83 & 28 & 83 & 28
\end{tabular}




\begin{tabular}{l|l|l|l|l} 
& \multicolumn{4}{|c|}{ ACCEPTED MANUSCRIPT } \\
N-120 & 100 & 27 & 100 & 26 \\
N-150 & 118 & 37 & 118 & 36 \\
N-200 & 164 & 30 & 165 & 27 \\
N-250 & 208 & 28 & 209 & 24 \\
N-300 & 250 & 27 & 248 & 26
\end{tabular}

Table 3. Doses delivered to quartz and feldspar grains in the different irradiation configurations. " $\overline{E_{\gamma}} "$ is the average photon energy for each radiation quality. " $\mathrm{K}_{\text {air }}$ " is the kerma in air as determined experimentally at the Laboratory for Nuclear Calibrations (LNK) of SCK.CEN, and " $\sigma_{\text {cal }}$ " its associated uncertainty. "Dose" is the dose received by the grains for a $1 \mathrm{~Gy}$ kerma in air irradiation. " $\sigma_{\text {stat }}$ " corresponds to the corresponding statistical uncertainty from the GEANT4 simulations of the irradiation setup, and " $\sigma_{\text {tot }}$ " to the total uncertainty, estimated as the quadratic sum of " $\sigma_{\text {stat }}$ " and " $\sigma_{\text {cal }}$.

\begin{tabular}{|cccc|ccc|ccc|}
\hline $\begin{array}{c}\text { Radiation } \\
\text { quality }\end{array}$ & $\begin{array}{c}E_{\gamma} \\
(\mathrm{keV})\end{array}$ & $\begin{array}{c}\mathrm{K}_{\text {air }} \text { rate } \\
(\mathrm{mGy} / \mathrm{h})\end{array}$ & $\sigma_{\text {cal }}$ & \multicolumn{3}{|c|}{ Quartz } & \multicolumn{3}{c|}{ Kose } \\
$(\mathrm{Gy})$ & $\sigma_{\text {stat }}$ & $\sigma_{\text {tot }}$ & Doseldspar \\
$(\mathrm{Gy})$ & $\sigma_{\text {stat }}$ & $\sigma_{\text {tot }}$ \\
\hline${ }^{137} \mathrm{Cs}$ & 662 & & & 0.94 & 0.01 & 0.02 & 0.94 & 0.01 & 0.02 \\
$\mathrm{~N}-10$ & 8 & 4510 & 226 & 1.60 & 0.00 & 0.08 & 1.88 & 0.00 & 0.09 \\
$\mathrm{~N}-15$ & 12 & 1395 & 70 & 3.42 & 0.01 & 0.17 & 4.58 & 0.01 & 0.23 \\
$\mathrm{~N}-20$ & 16 & 3720 & 186 & 4.39 & 0.01 & 0.22 & 6.08 & 0.01 & 0.30 \\
$\mathrm{~N}-25$ & 20 & 2007 & 100 & 4.92 & 0.01 & 0.25 & 7.00 & 0.01 & 0.35 \\
$\mathrm{~N}-30$ & 25 & 1034 & 52 & 5.11 & 0.01 & 0.26 & 7.51 & 0.02 & 0.38 \\
$\mathrm{~N}-40$ & 33 & 411 & 21 & 5.02 & 0.02 & 0.26 & 7.50 & 0.03 & 0.38 \\
$\mathrm{~N}-60$ & 48 & 536 & 27 & 4.01 & 0.03 & 0.20 & 5.86 & 0.04 & 0.30 \\
$\mathrm{~N}-80$ & 65 & 222 & 11 & 2.53 & 0.02 & 0.13 & 3.58 & 0.02 & 0.18 \\
$\mathrm{~N}-100$ & 83 & 208 & 10 & 1.68 & 0.01 & 0.08 & 2.19 & 0.01 & 0.11 \\
$\mathrm{~N}-120$ & 100 & 202 & 10 & 1.34 & 0.01 & 0.07 & 1.57 & 0.01 & 0.08 \\
$\mathrm{~N}-150$ & 118 & 1047 & 52 & 1.09 & 0.01 & 0.05 & 1.16 & 0.01 & 0.06 \\
$\mathrm{~N}-200$ & 165 & 281 & 14 & 1.01 & 0.01 & 0.05 & 1.04 & 0.01 & 0.05 \\
$\mathrm{~N}-250$ & 209 & 216 & 11 & 0.96 & 0.01 & 0.05 & 0.96 & 0.01 & 0.05 \\
$\mathrm{~N}-300$ & 248 & 212 & 11 & 0.94 & 0.01 & 0.05 & 0.93 & 0.01 & 0.05 \\
\hline
\end{tabular}

Table 4. Average efficiency of $x$-ray irradiations relative to gamma ${ }^{137} \mathrm{Cs}$ irradiations. "s.e." corresponds to the standard error of the mean, and includes the calibration uncertainty on kerma in air values; "RSD" corresponds to the relative standard deviation of beta equivalent doses ( $n=7$ samples), i.e. before normalisation by the response to gamma irradiations (hence, the $1 \%$ value appearing in the first line means that there is only $1 \%$ standard deviation in beta equivalent doses for the gamma irradiations).

\begin{tabular}{|cccc|}
\hline $\begin{array}{c}\text { Radiation } \\
\text { quality }\end{array}$ & Average & s.e. & RSD (\%) \\
\hline${ }^{137}$ Cs & 1 & N/A & 1 \\
$\mathrm{~N}-10$ & 0.100 & 0.022 & 57 \\
\hline
\end{tabular}




\section{ACCEPTED MANUSCRIPT}

\begin{tabular}{|cccc|}
\hline $\mathrm{N}-15$ & 0.061 & 0.015 & 64 \\
$\mathrm{~N}-20$ & 0.258 & 0.017 & 12 \\
$\mathrm{~N}-25$ & 0.346 & 0.020 & 7 \\
$\mathrm{~N}-30$ & 0.422 & 0.025 & 8 \\
$\mathrm{~N}-40$ & 0.628 & 0.035 & 6 \\
$\mathrm{~N}-60$ & 0.786 & 0.046 & 8 \\
$\mathrm{~N}-80$ & 0.891 & 0.062 & 13 \\
$\mathrm{~N}-100$ & 0.972 & 0.050 & 5 \\
$\mathrm{~N}-120$ & 1.035 & 0.055 & 5 \\
$\mathrm{~N}-150$ & 1.034 & 0.057 & 6 \\
$\mathrm{~N}-200$ & 1.032 & 0.055 & 4 \\
$\mathrm{~N}-250$ & 1.068 & 0.062 & 7 \\
$\mathrm{~N}-300$ & 1.007 & 0.055 & 4 \\
\hline
\end{tabular}

